\title{
DÉPENDANCE AUX IMAGES ET ÉLABORATION DE LA VIOLENCE
}

Florian Houssier

\author{
Florian Houssier \\ Psychologue \\ clinicien, \\ Psychanalyste, \\ Membre \\ du Collège \\ International de \\ l'Adolescence \\ (CILA), Professeur \\ en psychologie \\ clinique et \\ psychopathologie, \\ Laboratoire : \\ Unité Transversale \\ de Recherches : \\ Psychogenèse et \\ Psychopathologie, \\ Université Paris- \\ Nord, Sorbonne \\ Paris Cité.
}

RESUME : Dans cet article, l'auteur explore l'usage des images violentes dans un jeu vidéo en interrogeant la fonction métapsychologique des images à l'adolescence. A partir de l'exposé d'un cas clinique, une discussion s'ouvre sur la différence entre le retrait sur les objets internes et l'importance des rêveries adolescentes que le jeu vidéo prolongerait, au service du processus adolescent.

Mots clés : Image, violence, jeu vidéo, adolescence, retrait, rêverie.

ABSTRACT: Image dependence and elaboration of violence. The author explores the use of violent images in a video game, examining the meta-psychological function of the images in adolescence. From a clinical case, we discuss the difference between the withdrawal on the internal objects and the adolescent daydreams, prolonged by the video game helping on the adolescent process.

Keywords: Image, violence, video game, adolescence, withdrawal, daydream.

D ans le champ socio-médiatique, l'image est souvent désignée comme une source d'influence censée orienter voire provoquer les passages à l'acte les plus violents. Pourtant, une image seule ne pense pas, quel que soit son contenu. L'image peut faire écran, lorsqu'on met en avant sa violence ou ses effets supposés, c'est-à-dire le visible au détriment de ce qui ne se voit pas, les processus internes qui lui donnent sa fonction dans l'économie psychique du sujet.

A l'adolescence, le rapport à l'image interroge plus en profondeur les relations d'objet et les remaniements instanciels. L’objet désigné comme l'ennemi intime, le corps pubère (BIRRAUX, 1990) désormais source de toutes les désorganisations potentielles, s'articule avec les sentiments de perte d'objet, sentiments en lien avec la haine de la vie psychique 
(GUILLAUMIN, 2001). L’objet d'amour ou de haine peut être représenté par l'image via une multitude de substituts, montrant la dépendance du sujet à ses objets internes au moment où les enjeux de d'individuation et de différenciation deviennent cruciaux. Dans le même temps, la pulsionnalité sauvage qui assaille les adolescents provoque la reprise de mécanismes de défense tels que la projection ou l'ascétisme, souvent sollicités par les joueurs vidéos (VLACHOPOULOU, HADDOUK, HOUSSIER, 2014) : la projection des conflits psychiques via la médiation des jeux ; l'ascétisme comme défense contre ces pulsions encore trop colorées de fantasmes incestueux et parricides. Alors que les conflits identificatoires créent un champ de bataille interne, l'image peut nourrir ainsi les identifications ou créer un leurre en rupture avec le projet identificatoire. Autrement dit, les jeux vidéos peuvent être utilisés comme un moyen de mettre à distance les figures parentales en tant que marqueur de la différence des générations ; ou comme une voie d'entrée dans une forme de repli narcissique contre-identificatoire, désaffilié, ce que nous discuterons à propos du retrait. La question qui traverse cet article peut ainsi être formulée ainsi : le rapport à l'image est-il la création d'un espace de jeu et de rêverie, dans la continuité de l'espace transitionnel développé par D. W. Winnicott ? Ou une addiction à l'image qui participe d'un mouvement de retrait vis-à-vis du monde externe et de son représentant central, l'autre différencié ?

La rêverie est généralement associée à une continuité du jeu chez l'enfant ; à l'adolescence, elle participe notamment de la capacité à différer la conquête de l'objet sexuel non incestueux et extra-familial (FREUD, 1905/1962). A contrario, le retrait dans une rêverie oniroïde est souvent envisagé comme un dispositif anti-dépressif s'opposant à l'élaboration des désirs liés à l'Édipe pubertaire (GUTTON, 1996). Les extraits de psychothérapie que nous présentons interrogent l'articulation entre image de jeu vidéo et rêverie, posant le débat en ces termes : l'usage de l'image en tant qu'objet culturel est-il addictif, participant d'une impasse dynamique du processus d'adolescence ? Ou cet usage, aussi compulsif soit-il, est-il lié à la capacité de rêverie caractérisant les transitions nécessaires à l'adolescent, temps d'attente au service de l'élaboration des conflits infanto-adolescents ?

\section{L'USAGE DES IMAGES, ENTRE DOUBLE NARCISSIQUE ET DÉCORPORATION}

Le virtuel, le monde des images n'est pas à incriminer en soi. C'est même un support de construction de soi lorsqu'il s'inscrit dans la créativité, l'espace d'illusion. Comme le rêve, il passe par la figurabilité, le déplacement, la condensation et la symbolisation. C'est la dépendance à ce type de moyen que trouve l'adolescent qui est en cause, la difficulté et l'angoisse majeures étant pour lui 
précisément d’assumer sa métamorphose et sa nouvelle solitude face aux autres adultes et pairs. Que ce soit à partir du jugement qu'il porte sur lui-même et son image, ou le poids du regard des adultes dans son environnement, l'image touche les mondes internes comme externes. Les adolescents ont à assumer une transformation traumatique en même temps que le regard que portent les autres sur cette transformation.

Deux exemples de l'usage des images peuvent être convoqués. L'image est d'abord surface sensible avant de devenir support de sens. Elle est support de projection du monde interne avant d'être regardée et interprétée, perçue et relue comme une réalité externe qui donnerait en quelque sorte des nouvelles de soi-même. Ainsi, le « dialogue » avec son avatar virtuel peut être pensé comme une figure du double narcissique, aussi triomphant dans le jeu que l'adolescent peut se sentir impuissant face à l'intensité affolante des conflits pubertaires.

L'adolescent se regarde dans l'image qu'il perçoit et qu'il redessine mentalement. Elle est autant figuration d'un état interne quappel à représentation, appartenant autant à la réalité externe qu’elle est écho réverbérant et créatif du monde interne. Ce sont ces entrelacs qui donnent à l'image autant de poids pour l'adolescent.

A contrario, cette sensibilité au percept conduit certains adolescents à rechercher les images qu'ils produisent sous l'effet de toxiques. Ces images sous influence cannabique voire sous hallucinogène, offrent un certain plaisir esthétique et sensoriel en même temps. Comme les images du rêve, ces productions hallucinées condensent une multitude de contenus, donnant la sensation à celui qui les produit d'une grande facilité créatrice dont il est pourtant bien difficile de parler ensuite. La rapidité et la fugacité des perceptions procurent une impression particulière que ces adolescents recherchent ensuite dans les effets spéciaux de certains jeux vidéo. La recherche de perception hallucinée d'images et de sensations mêlées montre à quel point le fonctionnement psychique de l'adolescent sollicite les processus primaires. L'image, dans ce cas, n'est pas investie comme support de représentation et de sens mais est recherchée comme porteuse de sensations (MARTY, 2009).

A l’adolescence, la figure de l'anorexie mentale représente de façon paradigmatique la tentative de nier les changements psycho-corporels de l'adolescence ; nier le corps passe par une « décorporation » (HOUSSIER, 2011) qui, souvent à bas bruit, est présente dans l'usage intensif de jeux vidéos : nuits blanches passées à jouer sans boire ni manger, surinvestissement des images recouvrant, à la façon d'un souvenir-écran, d’autres représentations. Un des enjeux du rapport aux images est celui qui traverse le processus adolescent : l'envers de cette décorporation relève de l'intégration de la violence d'un nouveau corps, cette fois définitivement sexué ou en voie de l'être. 


\section{RENAUD, DE L'ÉCRAN MATERNEL À LA RENCONTRE AVEC L'OBJET}

«Quand mon père est mort de son cancer des os, j’avais sept ans. D’apprendre aujourd'hui que quand il s'enfermait dans sa chambre, c'était pour se shooter, ça casse l'image ».

Tels sont les propos tenus par Renaud, âgé de dix-sept ans, dans le cadre de sa séance hebdomadaire de psychothérapie. Depuis le début de son adolescence, l'image a pris une place décisive dans sa vie, objet de conflits avec sa mère. Lorsqu'elle apprend qu'il ne va plus en cours et reste à la maison pour jouer à «World of Warcraft » en attendant son retour, les disputes éclatent ; reprenant l'aspect passionnel dont il a gardé quelques souvenirs et dont ses sœurs lui ont parlé, les scènes de dispute ressemblent à des scènes de ménage, rappelant les conflits entre les parents avant la mort du père. Un monde familial en guerre, pourrait-on ajouter en écho au titre du jeu vidéo ; notons à ce propos que ce jeu fait partie de ce qu'on appelle les jeux d'Heroic Fantasy, fantasy pouvant être traduit par «fantasme » notamment. Le caractère héroïque de ce monde fantastique rappelle le triomphe teinté de mégalomanie des rêveries adolescentes.

Toujours est-il que, dans l'actuel, la mère de Renaud finit par lui supprimer son écran, forme de castration à la hauteur de l'intensité de l'investissement des images-écran ; celles-ci viennent raviver les fantasmes de rivalité avec la mère toute-puissante des premiers temps, celle qui grâce aux traductions/interprétations qu'elle propose à son enfant, est la principale pourvoyeuse d'images non créées par le bébé. A la place du jeu vidéo, d’autres images provoquées interviennent lorsque Renaud commence à fumer du cannabis. « Je joue toujours en réseau, avec les autres, ce n’est pas de l'isolement », dit-il comme pour se justifier par rapport aux reproches maternels mais aussi pour m'en convaincre. Dans ce jeu, il est question de passer des niveaux, pour explorer et trouver de nouveaux territoires. Ce jeu exploratoire lui fait oublier sa tristesse, le relie à sa mère - rester chez sa mère pour jouer, sur fond de phobie scolaire — - alors qu'il le sentiment de perdre du terrain dans leur relation. Jouer en « séchant » les cours lui permet de faire remarquer son absence dans une double identification : au père mort, à la mère insuffisamment présente. «C'est déprimant de penser que lorsque je vais en cours, je vis ça comme un abandon de la part de ma mère, je n'avais pas vu ça sous cet angle », dit-il suite à une interprétation.

Venir aux séances, c'est aussi se priver de ce ressort libidinal : se mettre en absence pour mieux mobiliser et recevoir l'inquiétude et la sollicitude, le holding source de ravitaillement affectif. Il manque régulièrement ses séances, tout étant d'accord, avec sa mère et moi, pour les payer. L'ordinateur est le lieu du conflit avec la mère, mais aussi un moyen d'exciter le lien à sa mère. Ou encore, le moyen de « se changer les esprits », ce qui m’évoque l'image d'un père qui reviendrait le hanter. Il protège coûte que coûte l'image de son père, 
trop endommagée pour qu'il puisse le supporter. A la suite d'une moquerie d'un lycéen sur son père, il passe trois jours à jouer sur internet sans dormir, « pour oublier », m’annonce-t-il. L'image dévalorisée du père contraste avec le fait qu'il passe des niveaux à son jeu vidéo, qu'il avance triomphalement en surmontant tous les obstacles de son jeu.

Mais voilà : la tentation du repli sur le monde des images cède maintenant la place à autre chose, ça bouge. Il est très fier, après tant d'hésitations et de conflits — rester avec ma mère ou rencontrer les autres au lycée ? —, d’avoir des relations sexuelles avec une fille. L'image a fait écran jusqu'ici, mais la rencontre avec l'objet sexuel s'offre à lui, ouvrant sur une potentielle complémentarité des sexes. « Si je fixais un classement affectif, je mettrais mes deux sœurs en premier, ma copine, je ne sais pas si j'inclurais ma mère dans ce classement, on a une relation trop aléatoire ». Sa mère a rencontré un nouvel amant, elle n'est plus là quand il rentre du lycée. « Je me demande ce que je ressentirais si elle mourrait. Je serais sans doute triste à long terme, mais pas sur le moment, on n'a plus la même relation qu'avant. » Puis, à la suite de séances qu’il manque, je repère avec lui qu'en s'absentant, il maîtrise la relation, décide quand il vient, tout en ne disant pas à sa mère qu'il manque les séances, me mettant dans une complicité transgressive avec lui. En même temps, dans le transfert, il me fait vivre la discontinuité de la relation avec son père.

«Mon rapport à l'absence est ce qu'on a trouvé de plus important ici, je crois ». Mais avec son amie, il n’arrive pas à s'absenter, il la colle, elle le lui reproche, elle rompt. Quand il rentre à la maison, il évite sa mère pour éviter les conflits, un trop grand rapprochement entre eux.

Pendant que l'ombre du père continue de circuler dans le lien, la relation à sa mère bascule. Pas de perte d'objet, mais plutôt un glissement le long d'une autre équation symbolique : de la mère à l'objet post-maternel, pas encore l'objet écran, celui du refoulement. Les zones de pensée sont encore trop proches, le lien encore trop direct entre sa mère et les filles qu'il rencontre. Un préconscient encore traversé par des trouées : se reprennent la jalousie, la demande d'exclusivité, l'omniprésence exigée vis-à-vis des filles qu’il rencontre. «Je n’arrive pas à oublier la première, c'est avec elle que c'était le mieux, y compris sur le plan sexuel ».

Il se désespère : «Avec les filles, j’ai découvert une vraie addiction »; sa première petite amie le quitte mais ils restent en contact et, après qu'elle a flirté avec un autre adolescent, il la reconquiert. Les reproches à la mère sont maintenant adressés à son amie ; elle se ment à elle-même, elle ne veut pas voir la réalité en face, elle le trompe avec un autre. Il s'interroge sur son absence à certaines séances : non, me dit-il spontanément, je ne lui ai pas trop manqué. Parfois, il regrette de ne pas venir car il sait qu'il sera content d'être là, en ma présence. En entendant cet énoncé, j’associe pour moi-même ; je me sens comme une figure 
paternelle dans les coulisses : plutôt dans l'ombre, non intrusif et peu inscrit dans le réel, mais qu'il peut utiliser lorsque lui choisit de venir à sa séance alors que la demande était avant tout « portée » par sa mère.

Un déplacement a bien lieu, l'image n’est plus nécessaire pour éviter de penser et souffrir, la sexualité découverte recouvre l'image vidéo. L'image a été le tenant-lieu d'espace psychique auxiliaire, le temps de découvrir le plaisir et la conflictualité de l'addiction sexuelle et sentimentale, le temps de redécouvrir l'objet, celui de sa passion. Un nouvel espoir émerge : ne pas revivre l'abandon. « Tu te débarrasses de moi ! », reprochait-il à sa mère lorsqu'elle l'a envoyé en pension à la suite de son absentéisme scolaire. Il se coupe alors au niveau des bras, se désespère lorsqu'elle ne le remarque pas, malgré ses tee-shirts à manches courtes. L’angoisse d'abandon et la dépendance à l'objet sont maintenant représentées, mises en acte dans des liens vivants ; il ne ressent plus, si ce n'est à l'occasion de courts épisodes dépressifs, le désir de se replier sur un monde d’images.

\section{RÊVERIE ET ESPACE D'ILLUSION : AMÉNAGEMENT ET REMANIEMENT INTERNES}

S. Freud (1908/1985) situe la rêverie dans le droit fil du jeu infantile, dont il constitue un substitut. La rêverie corrige une réalité insatisfaisante par la construction fantasmatique de scénarios érotiques ou ambitieux. Les rêveries ne deviennent une voie latérale qui mène à la pathologie que lorsqu'elles deviennent foisonnantes, investies comme des satisfactions fantasmatiques se substituant à la relation sexuelle.

Se répandre en fantasmes, écrivait $\mathrm{S}$. Freud à propos de l'adolescent qui patiente avant la rencontre de l'autre sexe. Consommer de l'image, c'est attendre en donnant une place centrale à l'espace de la rêverie. Pour Renaud, la violence de l'arrachement à la mère et les conflits qu'il mobilise renvoie à la violence des combats mis en jeu par les images. Le contenu des jeux représente souvent des scènes héroïques ou érotiques qui voient l'adolescent triompher de tous les obstacles : on peut y lire le souhait d'être l'enfant idéal oedipien, sous-tendu par la supériorité sur le rival. Les conflits internes trouvent dans le jeu vidéo une surface de projection externe (HOUSSIER, 2009), à la fois familière dans sa capacité de contenance, et suffisamment à distance quant aux contenus exposés. L'adolescent méconnait la destructivité adressée à l'objet primaire qui se rejoue dans le monde des images ; de même, même si le terme nécessiterait une utilisation prudente au vu de la polysémie de définitions qui le caractérise aujourd'hui, l'identification projective n'est pas absente : si on suit le fil de l'identification quasi-adhésive au personnage incarné dans le jeu, celui-ci est vécu par l’adolescent comme animé de l'intérieur par ses désirs, évoquant à propos de la façon dont le nourrisson envahit l'objet primaire pour le contrôler de l'intérieur. 
Dans un jeu vidéo centré sur des enjeux de conquête, le plaisir pris au spectacle de la destruction est relié au fait que voir est toujours absorber et détruire ; destruction d'autant plus supportable dans l'inconscient qu'elle est sous-tendue par une conviction toute-puissante : nous pouvons recréer l'univers par un simple coup d'œil (BONNET, 1981). Les traces, les résidus psychiques sollicités par l'image fonctionnent comme un mobilisateur du paysage interne : en tant qu'effet miroir, l'image montre la destruction de l'objet rival, ou la séduction de l’objet de désir.

Jouer avec les images relève de la création d'un espace de maîtrise des objets internes, à l’aide d’un média. Pour Renaud, cela consiste à attendre, encaisser l'impact traumatique de l'adolescence, avant de pouvoir passer du virtuel au réel de l'objet (HOUSSIER, 2008). Par analogie, on peut comparer la rêverie à un jeu avec des images, comme le jeu vidéo à une forme de rêverie : dans les deux cas, il est question d'exploration de mondes potentiels à l'aide de représentants d'objet, dans un espace psychique aux potentialités étendues. Dans ce temps de transition, l'espace d'illusion maintient le fantasme que tout reste possible. Le sentiment de maîtrise que permet l'utilisation du jeu nourrit une illusion omnipotente encore nécessaire ; cet espace d’illusion rappelle le cheminement du nourrisson confronté à la tension entre voie hallucinatoire de satisfaction et prise en compte du principe de réalité.

Passer par des moments régressifs implique une expérimentation en fantasme avant le passage à l'action. La continuation du jeu et de l'espace de rêverie est une expérimentation agie virtuellement, une action d'essai préparatoire, le temps d'un travail d'élaboration, même partiel, des enjeux de perte qui émergent dans la psychothérapie. A la façon de la création d’un nouveau roman familial, le jeu vidéo revisite les origines pour les reformuler, les perlaborer dans un registre plus génitalisé.

L'usage intense des images externes renvoie à la lutte que l'adolescent mène contre la séparation avec ses objets internes, figures parentales incestueuses auxquelles il est question de renoncer. Le repli dans la rêverie - ou dans une de ses extensions, le jeu écran — ne traduit pas toujours un rejet du monde externe. Sa mise à distance témoigne de ce que l'adolescent lui attribue projectivement : angoisse de castration, frustration, impossibilité de se préserver ou de fuir des situations imaginées comme à haut risque anxiogène comme le permet la maitrise des attaques internes réinjectées dans le jeu ou que ce dernier remobilise. Dans la rêverie, la réalisation de désir infantile est conservée, même si cette satisfaction reste fictive, partielle.

La fuite du monde externe représente symboliquement une fuite de l'angoisse dépressive. La pente addictive vis-à-vis des jeux vidéo apparaît chez Renaud comme un temps dynamique, propice à l'élaboration progressive de l'angoisse 
de perte ; pour d'autres adolescents, l'usage de l'image s'inscrit dans un mouvement de retrait donnant une place prédominante à la sensorialité, au service de satisfactions partielles peu soumises au primat du génital.

\section{RETRAIT ET OBJETS INTERNES}

Dans le courant psychanalytique, adolescence et états-limites ont commencé à faire l'objet d'un profond travail d'élaboration au même moment, dans les années 50-60 (HOUSSIER, 2010). S’il est vrai qu'il se retrouve souvent dans les problématiques borderline ou psychotiques, le retrait est aussi propre à la névrose ou chez le sujet normal en période de tension. Davantage qu'un état, il s'agit d'une position constituant une forme de cachette libidinale, alternant de profonds moments de solitude et d'isolement avec des tentatives de contact avec le monde extérieur et les autres. La zone de calme offerte par le retrait est un repli dont souffre le patient, mais il peut être l'objet d'une idéalisation dans le sens du refuge, passant aussi par le désinvestissement du corps. Le retrait n’empêche pas de développer des relations, mais au détriment d'autres parties de la vie du sujet, elles bloquées. « Le retrait fait alors office d'espace mental où la réalité n’a pas à être affrontée, où le fantasme et la toute-puissance règnent sans partage, et où tout est permis. », ajoute J. Steiner (1996, p.18). Ces aspects rendent le retrait séduisant pour un adolescent, d'autant qu'il se sent à l'abri de l’angoisse, un espace calme et relativement protégé. Dans cette perspective, le retrait s'apparente à la retrouvaille avec une enveloppe de contenance restituant les limites du monde interne.

La fonction du retrait est comprise ici comme une défense contre toute élaboration de la position dépressive, sur fond de relation d'objet sado-masochique. Le retrait sert à se retirer pour gagner un sentiment de sécurité, en lien selon nous avec un « fantasme de garantie » (Houssier, 2013 a) dans le sens où, dans cette forme d'autosuffisance, une illusion est assurée et auto-alimentée, source de chaleur et de sécurité, le sentiment que tout est toujours possible et sans temporalité définie. Secrètement se maintient la conviction que tout peut redevenir possible, représentant le fantasme de permanence d'un lien de possession avec ses objets les plus archaïques.

Cependant, ces propositions sont à tempérer au regard des travaux de Winnicott. Il propose, en lien avec la capacité à être seul en présence de l'autre, une autre facette du retrait ; le retrait peut alors participer d'une capacité qui n'entraîne pas la perte de l'identification avec celui dont on s'isole (WINNICOTT, 1963/1970). Le retrait peut être aussi une communication en cul de sac qui aide le sujet à se sentir réel. C'est un état dans lequel la personne maintient une part régressée de son self et prend soin d'elle aux dépens des relations externes; le 
retrait ne soigne pas mais montre que le patient a dû tenir son self dans le passé ; le retrait est un appel à l'aide (WINNICOTT, 1954/1969) où le patient maintient le lien avec ses objets subjectifs ; cela témoigne alors d'une incapacité à être seul, dans un renversement de perspective par rapport à la présence de l'objet. Etre en retrait est une façon de maintenir le lien avec les objets internes, et non une voie d'accès pour se couper de ses objets.

Sortir du retrait revient à affronter sa réalité psychique en passant par le monde externe ; laisser partir l'objet signifie symboliquement le tuer ou le laisser mourir, enjeu central du processus d'adolescence. C'est la souffrance et la culpabilité, inséparables des confrontations avec la réalité, qui sont difficiles à tolérer et peuvent provoquer un retrait dans la toute-puissance, qui pourrait correspondre, dans un processus non psychotique, à ce que J. Steiner nomme « fermer un œil » (Ibid, p.190) ; fermer un œil correspond à la peur d'affronter sa vérité intérieure, donnant lieu à la dissimulation, la réalité étant alors faussement représentée et déformée par son travestissement. L'impuissance infantile est camouflée par l'éprouvé de toute-puissance dans le retrait, les figures de l'entourage étant trop faibles pour apporter de l'aide. La régression — pulsionnelle, relationnelle, motrice — est antagoniste à la mise en mot, jusqu'au début de la psychothérapie. Davantage que la capacité à fantasmer, c'est la possibilité de dire qui est remplacée par le repli. Il représente l'effort déployé par l’adolescent pour intérioriser un événement traumatique tout à la fois source potentielle de sens et porteur des achoppements de son histoire infantile (HOUSSIER, 2013 b).

\section{CONCLUSION}

Notre propos suggère un lien étroit entre l'usage de l'image et la rêverie à l'adolescence ; sauf que mettre des images entre les pensées latentes et conscientes revient à recréer un écran entre, une figure du préconscient. Le monde interne de l'adolescent est parfois trop dangereux pour laisser libre cours à la rêverie, pour se laisser aller à rêvasser, en contact avec ses conflits et représentations au moment où elles peuvent faire irruption. Ici, comme un théâtre interne prolongé dans le jeu vidéo, le jeu est un média qui sert de véhicule aux représentations ; il protège le lien oedipien tout en préservant de l'angoisse de castration liée à la rencontre avec l'objet sexuel extra-familial. Dans la dépression de l'adolescence, l'espace d'illusion maintient une relation passive à la mère tout en différant les enjeux de rivalité liés à la conquête de l'objet.

Les fantasmes adolescents relèvent de « représentations qui ne sont pas destinées à se réaliser » (FREUD, 1905). La dimension infantile du scénario fantasmatique choisi s'articule avec sa fixation par et dans l'activité fantasmatique, 
qui apparaît comme un des enjeux de l’adolescence : passer du désir agi dans le fantasme à celui réalisé avec l’objet.

Lorsque la libido narcissique perdure, cela fait signe d'une problématique de séparation où la rêverie devient représentative d'une fixation à un état narcissique prolongé. L'image, à la façon d'une rêverie, peut être au service d'une satisfaction substitutive érotisée, révélant l'impasse dans la rencontre à l'autre, préservant la relation avec l'objet maternel primaire mais aussi maintenant la bisexualité psychique de l'enfant perdure.

Recebido em 15/12/2013. Aprovado em 4/2/2014.

\section{RÉFÉRENCES}

BIRRAUX, A. (1990) L’adolescent face à son corps. Paris : Editions Universitaires. BONNET, G. (1981) Voir. Etre vu. Paris : PUF.

FREUD, S. (1905) Trois essais sur la théorie de la sexualité. Paris : Gallimard, 1962. . (1908) «Le créateur littéraire et la fantaisie », L'inquiétante étrangeté et autres essais. Paris : Gallimard, 1985, p.31-46.

GUILLAUMIN, J. (2001) Adolescence et désenchantement. Le Bouscat : L’esprit du temps.

GUTTON, P. (1996) Le pubertaire. Paris : PUF.

HOUSSIER, F. (2008) De l'image à la chair, Adolescence, 26, 4, p.891-892. (dir.) (2009) La violence de l'image. Paris : In Press.

(2010) L'école d'Anna Freud. Créativité et controverses. Paris: Editions Campagne Première.

(2011) « Positions psychotiques dans la cure d'une adolescente anorexique », in F. MARTY (dir.), Psychopathologie de l'adolescent : 10 cas cliniques. Paris : In Press, p.213-233.

. (2013 a) Meurtres dans la famille. Paris : Dunod.

(2013 b) «El adolescente, ese visitante de lo arcaic », Controversias on Line. Psicoanalisis de ninos y adolescentes (APdeBA), 12, p.58-71.

MARTY, F. (2009) «Images violentes à l'adolescence », in F. Houssier (dir.), La violence de l'image. Paris : In Press, p.49-69.

STEINER, J. (1996) Retraits psychiques : organisations pathologiques chez les sujets psychotiques, névrosés et borderline. Paris: PUF.

VLACHOPOULOU, X., HADDOUK, L., HOUSSIER, F. (2014) « Le héros virtuel comme révélateur de fantasmes archaïques à l’adolescence », Dialogue, 213, à paraitre.

WINNICOTT, D. W. (1954) «Repli et régression », in De la pédiatrie à la psychanalyse. Paris : Payot, 1969, p.223-230.

(1963) « De la communication et de la non-communication », in Processus de maturation chez l'enfant. Paris : Payot, 1970, p.151-168.

Florian Houssier

houssier.florian@gmail.com 